\title{
Methylwogonin exerts anticancer effects in A375 human malignant melanoma cells through apoptosis induction, DNA damage, cell invasion inhibition and downregulation of the mTOR/PI3K/Akt signalling pathway
}

\author{
Jiaorong Chen ${ }^{1}$, Chunmei Huang², Fangfang Liu², Zihui Xu³, Li Li², Zheng Huang², Hongfeng Zhang²
}

\begin{abstract}
${ }^{1}$ Department of Anatomy and Histology and Embryology, Basic Medical College, Hubei University of Traditional Chinese Medicine, Wuhan, China

2Pathology Department, The Central Hospital of Wuhan, Tongji Medical College, Huazhong University of Science and Technology, Wuhan, China

${ }^{3}$ Endocrinology Department, The Central Hospital of Wuhan, Tongji Medical College, Huazhong University of Science and Technology, Wuhan, China
\end{abstract}

Submitted: 5 November 2016

Accepted: 24 February 2017

Arch Med Sci 2019; 15 (4): 1056-1064

DOI: https://doi.org/10.5114/aoms.2018.73711

Copyright (c) 2018 Termedia \& Banach

\begin{abstract}
Introduction: The main purpose of the present research was to study the anticancer effects of methylwogonin in A375 human malignant melanoma cells by evaluating its effects on apoptosis, DNA fragmentation, cancer cell invasion and the mTOR/PI3K/AKT signalling pathway.

Material and methods: Effects on cell cytotoxicity were evaluated by MTT assay while a clonogenic assay determined the effects of methylwogonin on colony formation. Fluorescence microscopy evaluated apoptotic effects of methylwogonin in these cells using acridine orange/propidium iodide and Hoechst 33342 staining dyes. Gel electrophoresis evaluated the effects of methylwogonin on DNA fragmentation while the Matrigel invasion assay evaluated the effects of the drug on cancer cell invasion. Effects of methylwogonin on the mTOR/PI3K/AKT signalling pathway were evaluated by western blot assay.

Results: Methylwogonin induces concentration-dependent as well as timedependent growth inhibitory effects inducing significant cytotoxicity in these cancer cells. Methylwogonin led to dose-dependent inhibition of colony formation in A375 human malignant melanoma cells. The number of cell colonies decreased significantly as the methylwogonin dose increased from $0,50,150$, to $300 \mu \mathrm{M}$. Methylwogonin treatment of cells at lower doses led to yellow fluorescence (early apoptosis), which changed to red/orange fluorescence, indicating late apoptosis at higher doses. Similar results were obtained using Hoechst 33342 staining, revealing that 50, 150 and $300 \mu \mathrm{M}$ doses of methylwogonin led to significant morphological changes including chromatin condensation, fragmented nuclei and cellular shrinkage. DNA ladder formation was also observed, and this effect increased with increasing doses of methylwogonin. Methylwogonin also inhibited cancer cell invasion in a dose-dependent manner.

Conclusions: Different doses of methylwogonin led to concentration-dependent downregulation of phosphorylated PI3K, AKT and mTOR.
\end{abstract}

Key words: human melanoma, apoptosis, methylwogonin, fluorescence microscopy, cell invasion.

\author{
Corresponding author: \\ Hongfeng Zhang PhD \\ Pathology Department \\ The Central Hospital of Wuhan \\ Tongji Medical College \\ Huazhong University of \\ Science and Technology \\ Wuhan 430014, China \\ E-mail: hongfengzhang1234@ \\ hotmail.com
}




\section{Introduction}

The incidence and mortality rates for the majority of cancers are decreasing in most western countries, but in less developed and economically unstable countries they are increasing [1]. Melanoma is a type of cancer which develops from melanocytes [2]. Melanomas generally occur in the skin and are rarely found in mouth, intestines and eye. Exposure to ultraviolet light (UV) is one of the main causes of melanoma, and the risk of UV-induced melanoma is higher people with low levels of skin pigmentation [2]. The UV light may be from either the sun or from other sources, such as tanning devices [3]. Genetic defects such as xeroderma pigmentosum also increase the risk of melanoma. Surgery, radiotherapy, chemotherapy, immunotherapy and biotherapy are the treatment regimens for melanoma [4]. Melanoma is one of the most dangerous types of skin cancers. Globally, in 2012, it was detected in 232,000 people and resulted in 55,000 deaths. Australia and New Zealand have the highest rates of melanoma in the world. There are also high rates in Europe and North America, while it is less common in Asia, Africa, and Latin America. Melanoma is more common in men than women [4]. When the whole genomes of 25 melanomas were sequenced, an average of about 80,000 mutated bases, mostly C > T transitions and about 100 structural rearrangements, were found in each melanoma genome. This is much higher than the approximately 70 mutations across generations from parent to child [5].

Flavonoids are ubiquitous polyphenolic compounds present in plants and include an important part of the human diet all through the course of evolution. More than 4,000 distinct, natural flavonoids have been recognized thus far and have been consumed as part of common foods, wines, teas, traditional medicines and pharmaceutical drugs [6]. They exhibit properties valuable for human health because they interact with several cellular targets. Flavonoids show anti-oxidant and free-radical scavenger activities along with anti-inflammatory, antiviral, and particularly anticancer properties [7]. Chemoprevention of cancer using either natural compounds or synthetic materials has now become a very important issue in the management of carcinogenesis. Wogonin is an O-methylated flavone, a flavonoid-like chemical compound which has been isolated from Scutellaria baicalensis. The glycosides of wogonin are known as wogonosides [8]. Preliminary in vitro studies have shown pharmacological effects indicating that wogonin may have anti-tumour properties [9, 10]. Wogonin has also been found to possess anticonvulsant effects. It acts as a positive allosteric modulator of the benzodiazepine site of the $G_{A B A}$ receptor [11]. The main purpose of the current research work was to evaluate the anticancer effects of methylwogonin in A375 human melanoma cells and to investigate its effects on apoptosis, DNA fragmentation, cell invasion and the PI3K/Akt signalling pathway.

\section{Material and methods}

\section{Chemicals and other reagents}

Methylwogonin ( $\geq 95 \%)$, MTT (3-(4,5-dimethylthiazol-2-yl)-2,5-diphenyltetrazolium bromide), and dimethyl sulfoxide were obtained from Sigma-Aldrich Chemical Co. (St. Louis, MO, USA). Acridine orange, propidium iodide and Hoechst 33342 were procured from Wuhan Boster Biological Technology Ltd. (Wuhan, China). Dulbecco's modified Eagle's medium and RPMI-1640 medium were obtained from Gibco Life Technologies, Grand Island, NY, USA). Heat-inactivated fetal calf serum, penicillin, and streptomycin were obtained from Thomas Scientific, High Hill Road, Swedesboro, U.S.A.

\section{Cell line and cell culture conditions}

A375 human malignant melanoma cells were purchased from the cell bank of the Basic Medical College of Huazhong University of Science and Technology (HUST). The cells were maintained in DMEM supplemented with $10 \%$ FBS and antibiotics $(100 \mathrm{U} / \mathrm{ml}$ penicillin $\mathrm{G}$ and $100 \mu \mathrm{g} / \mathrm{ml}$ streptomycin) at $37^{\circ} \mathrm{C}$ in a humidified incubator.

\section{MTT assay for cell proliferation}

The cell cytotoxicity induced by methylwogonin was estimated by MTT cell viability assay using different doses of the drug and at different time incubations. Briefly, A375 human malignant melanoma cells were plated at a density of $1 \times 10^{6}$ cells per well in 96-well plates for $12 \mathrm{~h}$. The cells were then treated with $0,5,10,25,50,150$ and $300 \mu \mathrm{M}$ methylwogonin for 24 and $48 \mathrm{~h}$ periods. MTT solution $(20 \mu \mathrm{l})$ was added to each well. The medium was completely removed and $500 \mu \mathrm{l}$ of DMSO was added to solubilize MTT formazan crystals. The optical density was determined at $570 \mathrm{~nm}$ (OD570) using an ELISA plate reader (Model 550; Bio-Rad, Hercules, CA, USA).

\section{Clonogenic assay (colony forming assay) and determination of melanin content}

A375 human malignant melanoma cells $\left(2 \times 10^{6}\right.$ cells/well) were plated into a 6-well plate for adherence for $12 \mathrm{~h}$ prior to drug treatment. After the cells had adhered, the cells were subjected to the treatment of different doses $(0,50,150,300 \mu \mathrm{M})$ of methylwogonin for $48 \mathrm{~h}$. After this time inter- 
val, the used medium was discarded and the A375 cells were allowed to make colonies in complete medium for one week, after which colonies were fixed with acetic acid solution for 10 min, stained with Giemsa for $15 \mathrm{~min}$ and then the cells were counted manually under a light microscope. The melanin content of the melanoma cells was measured by the method as described previously by Hosoi et al. [12].

\section{Fluorescence microscopic study of apoptosis using acridine orange/ propidium iodide double staining}

The apoptosis induced by methylwogonin in A375 human malignant melanoma cells was assessed by a fluorescence microscope using a double staining dye (acridine orange/propidium iodide). The A375 cells were seeded in 6-well plates at a density of $2 \times 10^{5}$ cells/well. The cells were treated with increasing concentrations of methylwogonin $(0,50,150,300 \mu \mathrm{M})$ for $48 \mathrm{~h}$. Afterwards, the treated and untreated cells were incubated with acridine orange and propidium iodide (20 $\mathrm{\mu g} / \mathrm{ml}$ each) for $1 \mathrm{~h}$. The cell morphology was finally examined under a fluorescence microscope (Olympus IX 81, Tokyo, Japan) at 400× magnification.

\section{Fluorescence microscopic study}

\section{of apoptosis using Hoechst 33342}

The A375 human malignant melanoma cells were treated with increasing concentrations $(0,50$, $150,300 \mu \mathrm{M})$ of methylwogonin and the cells were placed in a $\mathrm{CO}_{2}$ incubator for $48 \mathrm{~h}$ at $37^{\circ} \mathrm{C}$. After incubation, the cells were fixed with $2.5 \%$ formaldehyde for 30 min and washed with PBS twice. The solution of Hoechst 33342 was added to the cells and after $20 \mathrm{~min}$ of staining the cells were observed under a fluorescence microscope at 200× magnification (Olympus IX 81, Tokyo, Japan).

\section{DNA fragmentation analysis}

In brief, A375 human malignant melanoma cells were seeded in a $60-\mathrm{mm}$ cell culture plate, incubated for $48 \mathrm{~h}$ and then treated with 0,50 , 150 , and $300 \mu \mathrm{M}$ of methylwogonin for $48 \mathrm{~h}$. Subsequently the A375 cells were harvested and washed twice with PBS before the pellets were lysed with a DNA lysis buffer for $30 \mathrm{~min}$. The sample was centrifuged at 12,000 rpm and the supernatant was prepared in an equal volume of $3.5 \%$ sodium-dodecyl sulphate, then incubated with $10 \mathrm{mg} / \mathrm{ml}$ RNase A for $2 \mathrm{~h}$. After adding $10 \mathrm{M}$ ammonium acetate, the DNA was precipitated with ethanol and collected by centrifugation at 12,000 rpm for 30 min. Finally, the DNA was dissolved in gel loading buffer, separated by electrophoresis in
3.5\% agarose gel, stained with ethidium bromide and examined under UV.

\section{Invasion assay}

After the A375 human malignant melanoma cells were washed with PBS, the cells were again suspended in serum free medium. Then $200 \mu \mathrm{l}$ of cell suspension $\left(2 \times 10^{6}\right.$ cells $\left./ \mathrm{ml}\right)$ was added to the upper chamber coated with Matrigel membrane (Millipore, Billerica, MA, USA) while the lower chamber was filled with medium containing $5 \%$ FBS. The cells were incubated with increasing concentrations $(0,50,150$, and $300 \mu \mathrm{M})$ of methylwogonin for $50 \mathrm{~min}$ at $25^{\circ} \mathrm{C}$. After $48 \mathrm{~h}$, the cells which still remained in the upper face of the filters were removed, and the cells which had travelled to the lower face of the filters were fixed with $90 \%$ methanol and then stained with $0.35 \%$ crystal violet and then counted using a light microscope.

\section{Determination of melanin content}

\section{Western blot analysis}

Next we used western blot assay to determine the main proteins involved in the anticancer effect produced by methylwogonin. A375 human malignant melanoma cells were harvested and lysed with RIPA buffer and the collected protein samples were examined using a bicinchoninic acid (BCA) protein assay kit for protein estimation. About $200 \mu \mathrm{g}$ of cellular protein from each sample was applied to 8-10\% SDS-polyacrylamide gels and probed with specific antibodies followed by exposure to horseradish peroxidase-conjugated goat anti-mouse antibodies. Blots were then developed using the West Pico Chemiluminescent substrate (Pierce; Woburn, MA, USA). The antibodies against $\beta$-actin, PI3K, Akt, p-PI3K, mTOR, p-mTOR, Bax, Bcl-2, and Cyt-c were obtained from Santa Cruz Biotechnology (Santa Cruz, CA, USA).

\section{Statistical analysis}

All results were depicted as mean \pm standard error (S.E.M) from at least three independent experiments. The differences between groups were analysed by one-way ANOVA. Significance of differences was shown as ${ }^{*} p<0.05,{ }^{* *} p<0.01$. The GraphPad prism 7 software was used for carrying out the statistical analysis.

\section{Results}

Methylwogonin induces potent cytotoxic effects in A375 human malignant melanoma cells

The fact that methylwogonin (Figure 1) induces strong cytotoxic effects in A375 human malignant 
<smiles>COc1cc(O)c2c(=O)cc(-c3ccccc3)oc2c1O</smiles>

Figure 1. Chemical structure of methylwogonin

melanoma cells was evaluated by MTT assay and the results are shown in Figure 2. As indicated in the figure, methylwogonin induces concentration-dependent as well as time-dependent growth inhibitory effects inducing significant cytotoxicity in these cancer cells. The $\mathrm{IC}_{50}$ values which were calculated at different incubation times were found to be 72.9 and $54.2 \mu \mathrm{M}$ at 24 and $48 \mathrm{~h}$, respectively. The half maximal inhibitory concen-

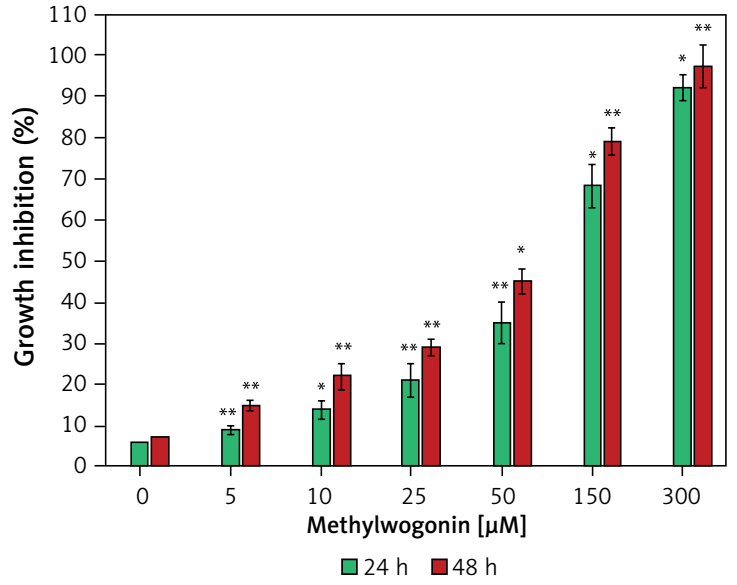

Figure 2. Growth inhibitory effects of methylwogonin in A375 human malignant melanoma cells. The cells were treated with increasing doses of methylwogonin and incubated for 24 and $48 \mathrm{~h}$

Data are shown as the mean $\pm S D$ of three independent experiments. ${ }^{*} p<0.05,{ }^{* *} p<0.01$, vs $0 \mu \mathrm{M}$ (control).
A

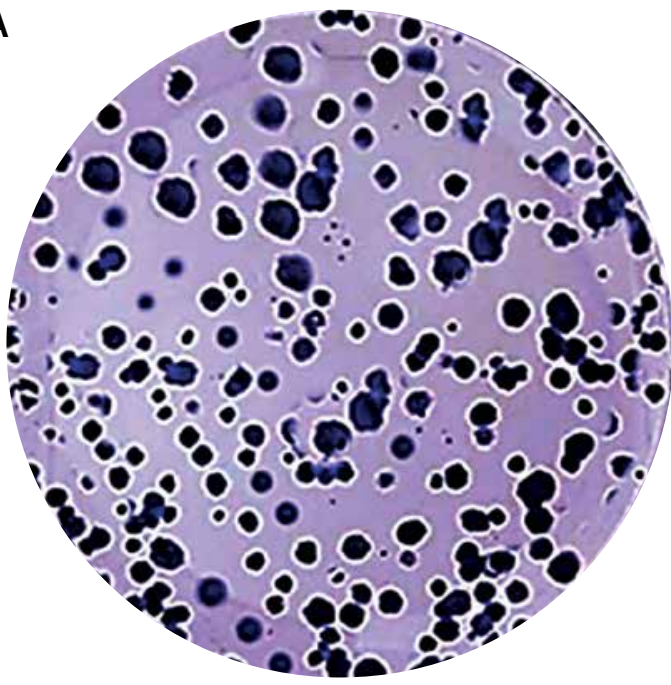

C

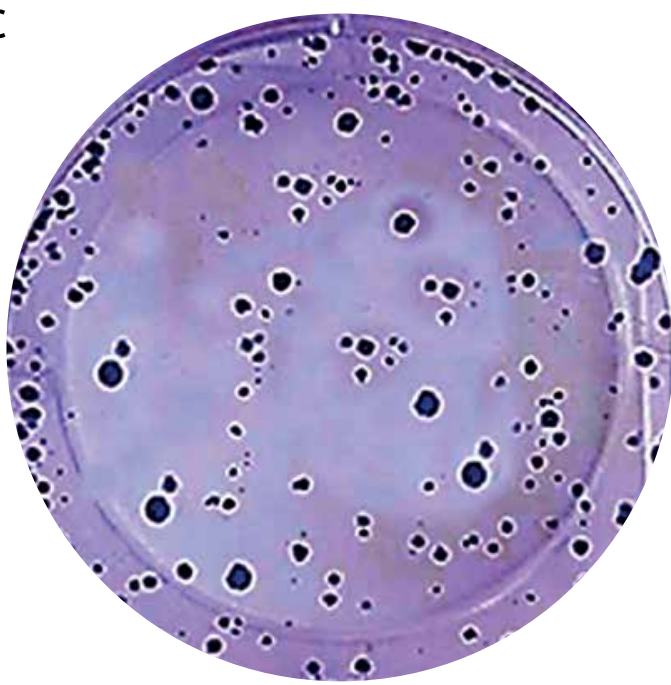

B

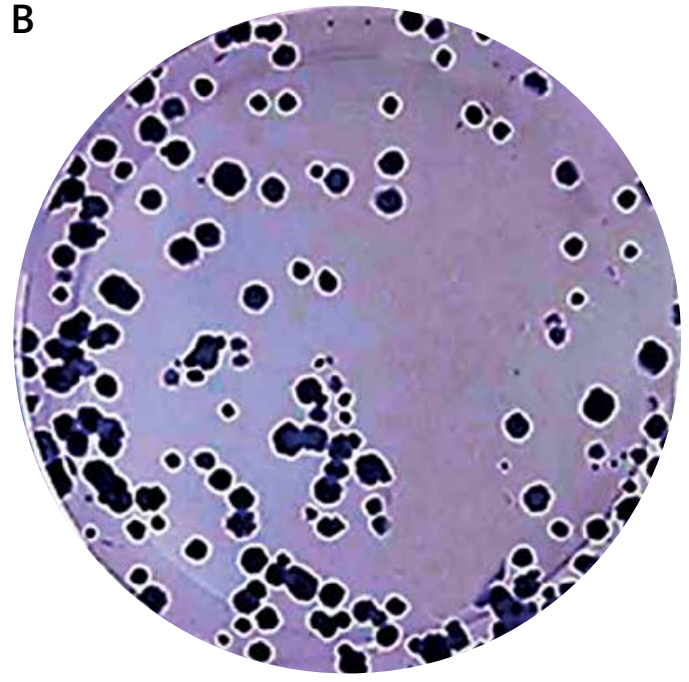

D

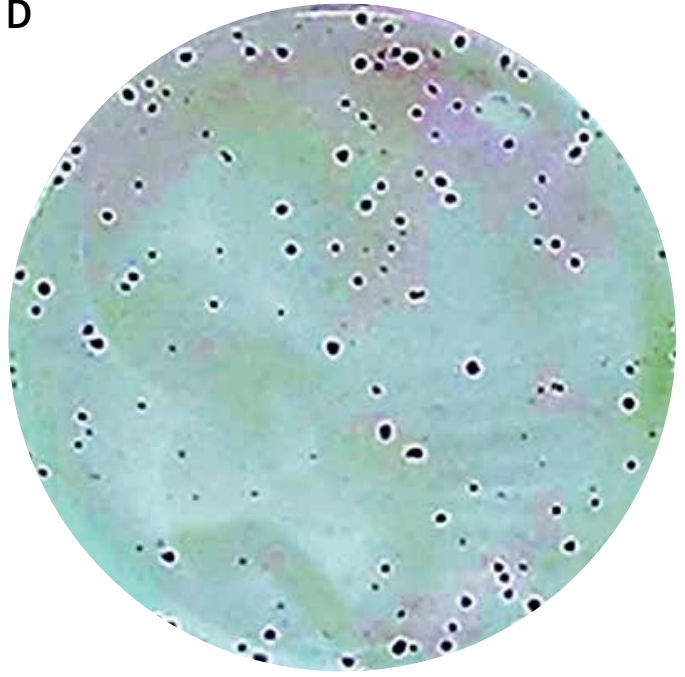

Figure 3. Inhibition of colony formation in A375 human malignant melanoma cells after treatment with 0 (A), 50 (B), 150 (C) and 300 (D) $\mu \mathrm{M}$ doses of methylwogonin for $48 \mathrm{~h}$ 


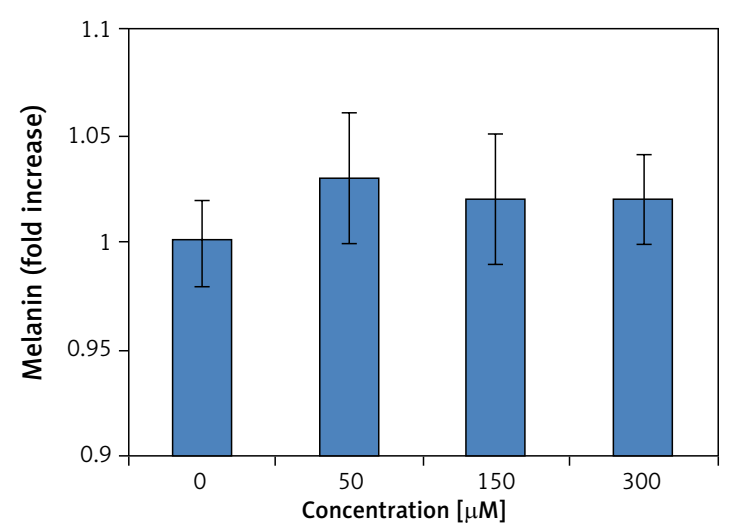

Figure 4. Melanin content in the A375 cancer cells colony formation

tration $\left(\mathrm{IC}_{50}\right)$ is a measure of the effectiveness of a substance in inhibiting a specific biological or biochemical function. Complete cell growth inhibition was observed at $48 \mathrm{~h}$ incubation with a $300 \mu \mathrm{M}$ dose of the drug.

\section{Methylwogonin led to inhibition of A375 cancer cell colony formation}

In order to evaluate whether methylwogonin induced both growth inhibitory effects, we used a clonogenic assay in order to assess effects of methylwogonin on the colony formation tendency in these cells. The results of this assay are depicted in Figure 3 and indicate that methylwogonin led to dose-dependent inhibition of colony formation in A375 human malignant melanoma cells. The number of cell colonies decreased significantly as the methylwogonin dose increased from 0 , 50,150 , to $300 \mu \mathrm{M}$. The colonies decreased by 47 , $64,78 \%$ at the concentrations of $50,150,300 \mu \mathrm{M}$ $(p<0.05)$ methylwogonin as compared to the untreated control. Thus taking both MTT and clonogenic assays into consideration, it is evident that methylwogonin induces cytotoxic effects along with inhibiting the colony formation tendency in these cells. Moreover, we also evaluated the melanin content of the cells, and no changes in melanin content were observed in the A375 cancer cells (Figure 4).

\section{Methylwogonin led to apoptotic induction in A375 human melanoma cells}

Further experiments were devised in order to evaluate the effects of methylwogonin on apoptotic induction in these cells using fluorescence microscopy with acridine orange/propidium iodide and Hoechst 33342 staining agents. The results which are shown in Figures $5 A-D$ and 6 A-D indicate that the untreated control cells showed prominent green fluorescence, indicating their viable nature (Figure $5 \mathrm{~A}$ ). However, methylwogonin treatment of cells at lower doses led to yellow fluorescence (early apoptosis) which changed to red/orange fluorescence at higher doses, indicating late apoptosis (Figures 5 B-D). Lower doses of methylwogonin induced early apoptosis while higher doses of the compound led to late apoptosis. The apoptotic cell populations increased from $2.5 \%$ in the untreated control up to $85 \%$ at $300 \mu \mathrm{M}(p<0.01)$ concentration of methylwogonin. Similar results were obtained using Hoechst 33342 staining, and the results are depicted in Figure 6 , indicating that untreated control cells showed normal cell morphology with no cell shrinkage. However, on treating cells with 50, 150 and $300 \mu \mathrm{M}$ doses of methylwogonin, several morphological changes in the cells including chromatin condensation, fragmented nuclei and cellular shrinkage occurred. Methylwogonin-treated cells exhibited bright fluorescence indicating rupture of the cell membrane and extensive staining (Figures 6 B-D).
A

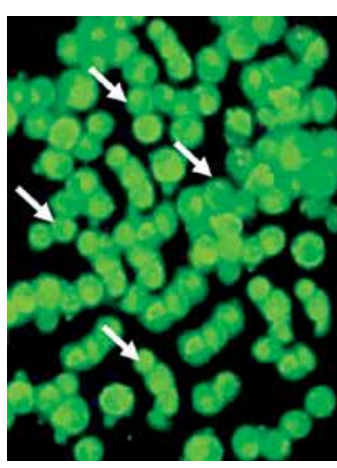

B

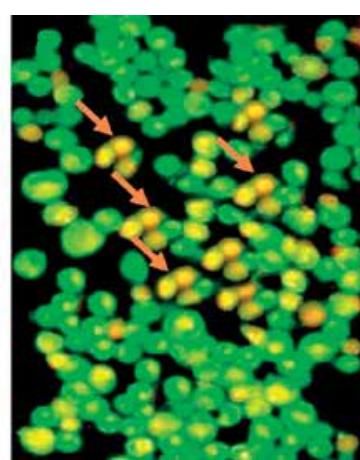

C

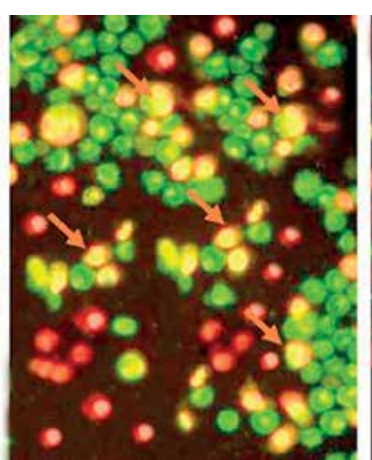

D

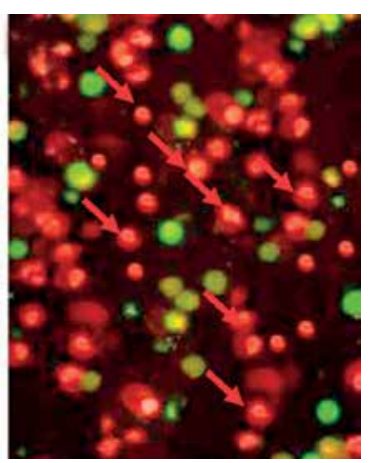

Figure 5. Fluorescence microscopic (400x magnification) study of the apoptosis induced by methylwogonin in A375 human malignant melanoma cells. The cells were treated with 0 (A), 50 (B), 150 (C), and $300 \mu \mathrm{M}$ (D) doses of methylwogonin for $48 \mathrm{~h}$ and then stained with acridine orange/propidium iodide double dye and finally examined by fluorescence microscope at $400 \times$ magnification. White arrows indicate viable cells, orange arrows indicate early apoptotic cells, red arrows indicate late apoptotic cells 
A

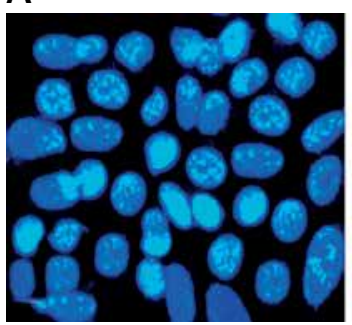

B

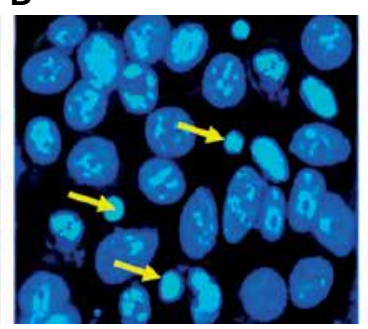

C

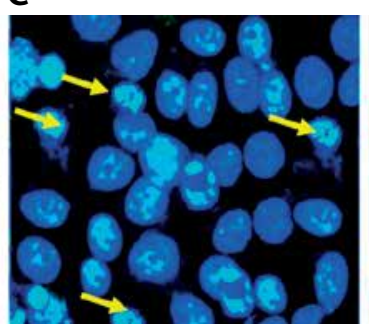

D

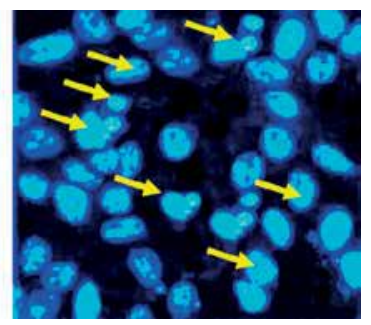

Figure 6. Fluorescence microscopic (at 200x magnification) study of apoptosis in A375 human malignant melanoma cells using Hoechst 33342 staining dye. The cells were treated with 0 (A, untreated cells), 50 (B), 150 (C) and 300 (D) $\mu \mathrm{M}$ concentrations of methylwogonin for $48 \mathrm{~h}$ and then stained with Hoechst 33342 before being analysed by fluorescence microscopy. Yellow arrows indicate cells with chromatin condensation, DNA fragmentation and cell shrinkage (characteristic of apoptosis)

\section{Methylwogonin led to DNA damage in A375 human melanoma cells}

DNA damage which was induced by methylwogonin in A375 human melanoma cells was examined using gel electrophoresis, which involves the formation of a DNA ladder. The findings of this assay are depicted in Figure 7 and indicate that DNA ladder formation was observed and this effect increased with increasing doses of methylwogonin. In the case of the untreated control group, no such DNA laddering was seen, indicating no DNA damage at all. The DNA fragmentation is believed to be a hallmark of the apoptotic process which initiates within the cell, further indicating that methylwogonin led to cell death through inducing apoptosis.

\section{Methylwogonin suppressed cell invasion in A375 human malignant melanoma cells}

The effect of methylwogonin on the cancer cell invasion in A375 human malignant melanoma cells was demonstrated by an invasion assay. The results, which are depicted in Figure 8 , indicate that methylwogonin also inhibited cancer cell invasion in a dose-dependent manner. The percent inhibition of cell invasion was reported to be up to $69 \%$ at $300 \mu \mathrm{M}(p<0.01)$ concentration. Che-

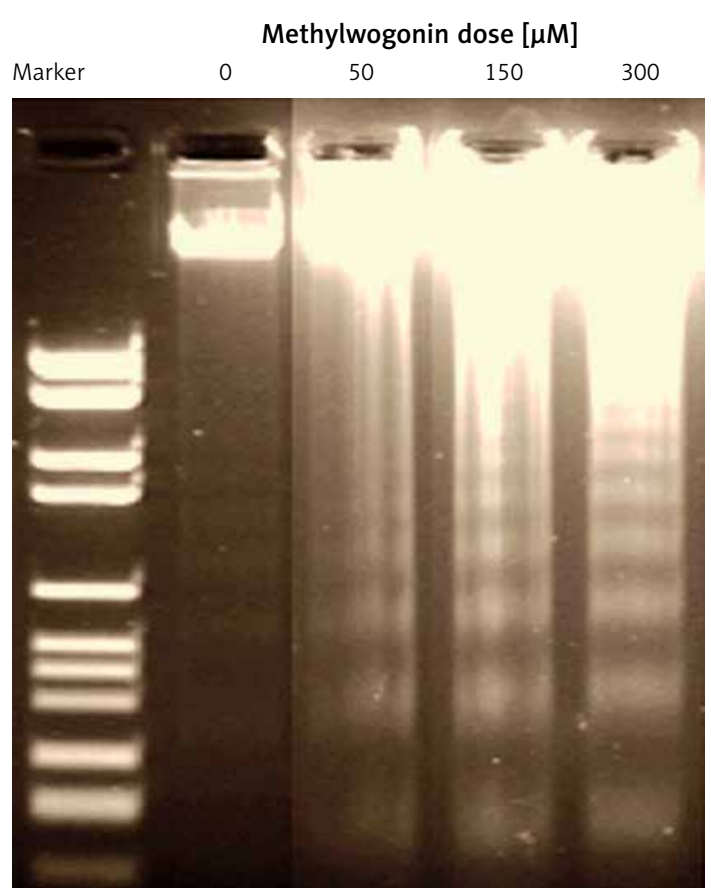

Figure 7. Methylwogonin induced DNA fragmentation in A375 human malignant melanoma cells. The cells were treated with 0 (control), 50, 150 and $300 \mu \mathrm{M}$ doses of the drug, incubated for $48 \mathrm{~h}$ and then harvested. The DNA was extracted and then analysed using 1.5\% agarose gel electrophoresis
A

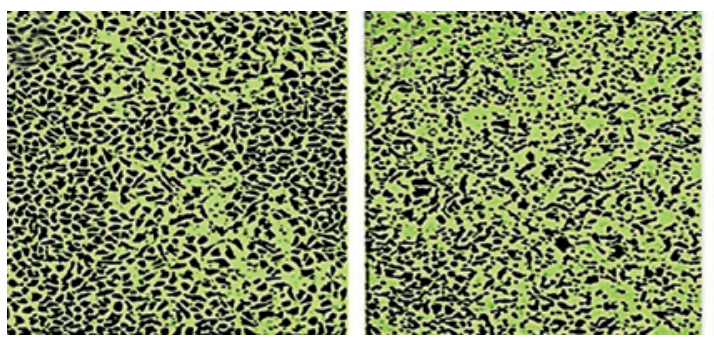

C

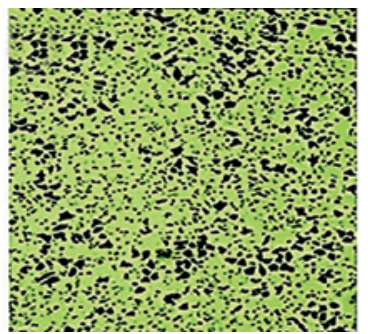

D

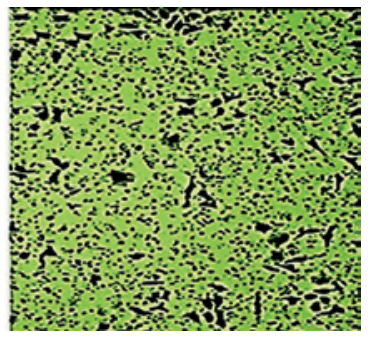

Figure 8. Inhibition of cell invasion in A375 human malignant melanoma cells. The cells were treated with 0 (A), 50 (B), 150 (C) and 300 (D) $\mu \mathrm{M}$ doses of methylwogonin for $48 \mathrm{~h}$ and the representative images were taken at $100 \times$ magnification 


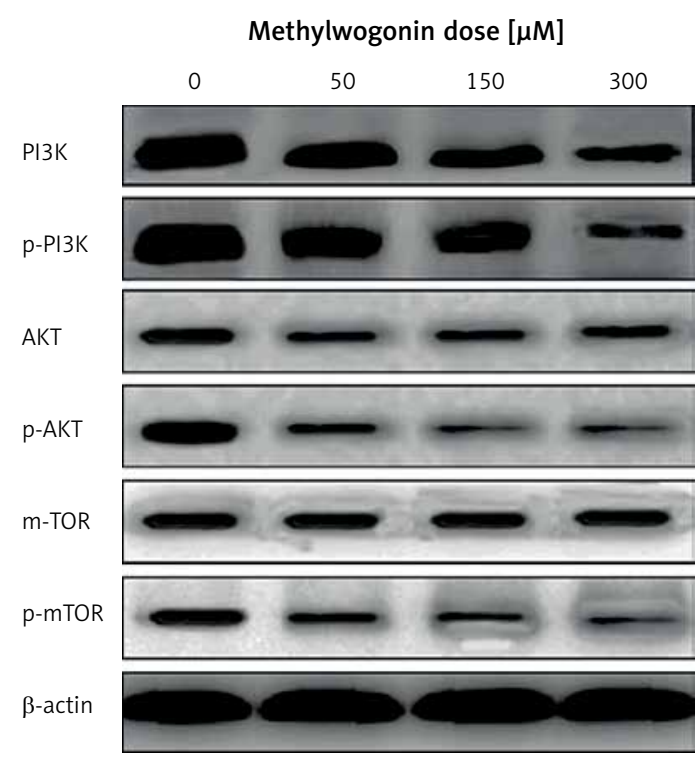

Figure 9. Methylwogonin leads to downregulation of PI3K/Akt signalling pathway in A375 human malignant melanoma cells. The cells were treated with $0,50,150$ and $300 \mu \mathrm{M}$ doses of methylwogonin for $48 \mathrm{~h}$ and the protein expression was evaluated by western blot. $\beta$-Actin served as a positive control

motherapeutic agents which suppress cancer cell migration and invasion are believed to be promising antitumor drugs because cancer cell migration and invasion have a direct relationship with cancer metastases.

\section{Methylwogonin led to significant alteration of mTOR/PI3K/Akt signalling pathway}

It is well established that the PI3K/Akt pathway has a close relationship with cell proliferation and cell survival. Therefore, our further aim was to evaluate the effects of methylwogonin on this crucial biochemical pathway. The results are depicted in Figure 9, which reveal that treatment of A375 human melanoma cells with different doses of methylwogonin led to concentration-dependent downregulation of phosphorylated PI3K, AKT and mTOR. However, it was found that total protein levels of PI3K, AKT and mTOR were not affected so much. Thus it now becomes evident that methylwogonin inhibited malignant melanoma cancer cell growth by regulating the mTOR/PI3K/AKT signalling pathway.

\section{Discussion}

Cancer is the second leading cause of death in Europe and America after cardiovascular disease. Discovery and development of anticancer drugs are the main emphasis of several pharmaceutical companies as well as non-profit government and non-government organizations. For many decades now, identification of cytotoxic compounds has led to the development of anticancer treatments. However, due to the development of multidrug resistance and emergence of severe side-effects, the use of cytotoxic anticancer drugs has been linked with limited efficacy, especially at advanced stages of the disease $[11,12]$. There is an urgent need of such compounds which can selectively target cancer cells without causing too much damage to the normal cells. An ideal drug for the treatment of cancer is one which causes less harm to the normal cells. Induction of apoptosis may be useful in the therapy and management or prevention of cancer. Certain naturally occurring molecules induce apoptosis in cancerous cells but not in normal cells. Increasingly it has become obvious that apoptosis for many anti-tumour agents is an important mode of action [13-15].

Wogonin is an O-methylated flavone, which resembles flavonoids. It is extracted from $S$. ba icalensis. Several previously published reports indicate that wogonin induces cytotoxic effects in various cancer cells including breast, osteosarcoma and malignant $T$ cells via the induction of apoptosis and cell cycle arrest. It was also reported that up to a dose as high as $100 \mu \mathrm{M}$ there was no cytotoxicity towards normal cells, indicating that wogonin induces selective cytotoxicity in cancer cells [9, 16-18]. It has also been reported that wogonin induced apoptosis via reactive oxygen species (ROS) generation along with modulation of PI3K/Akt, Bax and Blc-2-related signalling pathways. Studies also indicated that wogonin induced cytotoxicity in human promyeloid leukaemic cells. When tested it was found that wogonin was neither genotoxic nor apoptogenic in mice at indicated doses $[19,20]$. Wogonin also led to induction of human glioma cell death, which was mediated by ROS generation, GPR78 and GRP94 expression, and increased activity of caspases, such as caspase- 9 and caspase-3, eventually leading to apoptosis. Wogonin also inhibited cell mobility and invasion by upregulating the metastasis suppressor maspin gene [21, 22]. In summary, these data provide novel insights into the chemoprotective effect of wogonin, the main active ingredient of the Chinese medicine Scutellaria baicalensis. To the best of our knowledge, there are no reports on the antitumour effects of methylwogonin, which is a methylated derivative of wogonin. Previous studies have reported that melanin possesses radioprotective and scavenging properties and its existence can affect the susceptibility to the therapy, as shown in in vitro and in vivo experiments [23-26]. Therefore, we evaluated the effect of the test compound on the melanin status of the A375 human malignant melanoma cells (Figure 4). The results indicated the melanin content of the cells 
remained uniform throughout the experiment in all tested cell samples, and therefore it may be inferred that melanin content had no significant effect on the anticancer activity of methylwogonin the A375 human malignant melanoma cells. Further, we also studied the antitumor effects of methylwogonin on apoptosis induction, DNA damage, cancer cell invasion and the mTOR/PI3K/Akt signalling pathway. The MTT assay indicated that methylwogonin induced concentration-dependent as well as time-dependent growth inhibitory cytotoxic effects in these cancer cells. Moreover, the results of the MTT assay were also complemented by the results of the colony formation assay as the number of cell colonies decreased significantly with the increase in methylwogonin dose from $0,50,150$, to $300 \mu \mathrm{M}$. Lower doses of methylwogonin induced early apoptosis while higher doses of the compound led to late apoptosis. Methylwogonin-treated cells showed evident signs of morphological changes characteristic of apoptosis including chromatin condensation, fragmented nuclei and cellular shrinkage. DNA ladder formation was observed on methylwogonin treatment and this effect increased with increasing doses of methylwogonin. Methylwogonin also inhibited cancer cell invasion in a dose-dependent manner. Previous studies have reported that the activation of the PI3K/AKT/ mTOR pathway plays a vital role in ovarian cancer tumorigenesis, progression and chemotherapy resistance $[27,28]$. Therefore we evaluated the effect of methylwogonin on this pathway. Treatment of A375 human melanoma cells with different doses of methylwogonin led to dose-dependent downregulation of phosphorylated $\mathrm{PI} 3 \mathrm{~K}$, AKT and mTOR. However, it was found that total protein levels of PI3K, AKT and mTOR were not affected so much.

In conclusion, the present study revealed that methylwogonin induced potent antitumor effects in A375 human malignant melanoma cells by inducing apoptosis, DNA damage, inhibition of cell invasion and downregulation of the mTOR/PI3K/ Akt signalling pathway.

\section{Conflict of interest}

The authors declare no conflict of interest.

\section{References}

1. Ferlay J, Soerjomataram I, Dikshit R, et al. Cancer incidence and mortality worldwide: sources, methods and major patterns in GLOBOCAN 2012. Int J Cancer 2015; 136: 5 .

2. Tudrej KB, Czepielewska E, Kozłowska-Wojciechowska M. SOX10-MITF pathway activity in melanoma cells. Arch Med Sci 2017; 13: 1493-503.
3. World Cancer Report 2014. World Health Organization. 2014. Chapter 5.14.

4. Azoury SC, Lange JR. Epidemiology, risk factors, prevention, and early detection of melanoma. Surg Clin North Am 2014; 94: 945-62.

5. Roach JC, Glusman G, Smit AF, et al. Analysis of genetic inheritance in a family quartet by whole-genome sequencing. Science 2010; 328: 636-9.

6. Middleton E, Kandaswami C, Theoharides TC. The effects of plant flavonoids on mammalian cells: implications for inflammation, heart disease, and cancer. Pharmacol Rev 2000; 52: 673-751.

7. Manthey JA, Grohmann K, Guthrie N. Biological properties of citrus flavonoids pertaining to cancer and inflammation. Curr Med Chem 2001; 8: 135-53.

8. Park HG, Yoon SY, Choi JY, et al. Anticonvulsant effect of wogonin isolated from Scutellaria baicalensis. Eur J Pharmacol 2007; 574: 112-9.

9. Lin CC, Kuo CL, Lee MH, et al. Wogonin triggers apoptosis in human osteosarcoma U-2 OS cells through the endoplasmic reticulum stress, mitochondrial dysfunction and caspase-3-dependent signaling pathways. Int J Oncol 2011; 39: 217-24.

10. Gao J, Morgan WA, Sanchez-Medina A, Corcoran O. The ethanol extract of Scutellaria baicalensis and the active compounds induce cell cycle arrest and apoptosis including upregulation of p53 and Bax in human lung cancer cells. Toxicol Appl Pharmacol 2011; 254: 221-8.

11. Hui KM, Huen MS, Wang HY, et al. Anxiolytic effect of wogonin, a benzodiazepine receptor ligand isolated from Scutellaria baicalensis Georgi. Biochem Pharmacol 2002; 64: 1415-24.

12. Hosoi J, Abe E, Suda T, Kuroki T. Regulation of melanin synthesis of B16 mouse melanoma cells by 1 alpha, 25-dihydroxyvitamin D3 and retinoic acid. Cancer Res 1985; 45: 1474-8.

13. Schwartsmann G, Winograd B, Pinedo HM. The main steps in the development of anticancer agents. Radiother Oncol 1988; 12: 301-13.

14. Hoekstra R, Verweij J, Eskens FA. Clinical trial design for target specific anticancer agents. Invest New Drugs 2003; 21: 243-50.

15. Hirano T, Abe K, Gotoh M, Oka K. Citrus flavone tangeretin inhibits leukaemic HL-60 cell growth partially through induction of apoptosis with less cytotoxicity on normal lymphocytes. Br J Cancer 1995; 72: 1380-8.

16. D'Amico, Anthony V, Gillies McKenna W. Apoptosis and a re-investigation of the biologic basis for cancer therapy. Radiother Oncol 1994; 33: 3-10.

17. Kaufman SH, Desnoyers S, Ottaviano Y, Davidson NE, Poirier GG. Specific proteolytic cleavage of poly (ADP-ribose) polymerase: an early marker of chemotherapy-induced apoptosis. Cancer Res 1993; 53: 3976-85.

18. Yu JS, Kim AK. Wogonin induces apoptosis by activation of ERK and p38 MAPKs signaling pathways and generation of reactive oxygen species in human breast cancer cells. Mol Cells 2011; 31: 327-335.

19. Lee DH, Kim C, Zhang L, Lee YJ. Role of p53, PUMA, and BAX in wogonin-induced apoptosis in human cancer cells. Biochem Pharmacol 2008; 75: 2020-33.

20. Lee WR, Shen SC, Lin HY, et al. Wogonin and fisetin induce apoptosis in human promyeloleukemic cells, accompanied by a decrease of reactive oxygen species, and activation of caspase 3 and $\mathrm{Ca}(2+)$-dependent endonuclease. Biochem Pharmacol 2002; 63: 225-36.

21. Attia SM, Ahmad SF, Harisa Gl, et al. Wogonin attenuates etoposide-induced oxidative DNA damage and 
apoptosis via suppression of oxidative DNA stress and modulation of OGG1 expression. Food Chem Toxicol 2013; 59: 724-30.

22. Dong $P$, et al. Wogonin, an active ingredient of Chinese herb medicine Scutellaria baicalensis, inhibits the mobility and invasion of human gallbladder carcinoma GBC-SD cells by inducing the expression of maspin. J Ethnopharmacol 2011; 137: 1373-80.

23. Yan H, Hou X, Li T, et al. CD4+ T cell-mediated cytotoxicity eliminates primary tumor cells in metastatic melanoma through high MHC class II expression and can be enhanced by inhibitory receptor blockade. Tumour Biol 2016; DOI: 10.1007/s13277-016-5456-5.

24. Valpione S, Moser JC, Parrozzani R, et al. Development and external validation of a prognostic nomogram for metastatic uveal melanoma. PloS One 2015; 10: e0120181.

25. Slominski A, Zbytek B, Slominski R. Inhibitors of melanogenesis increase toxicity of cyclophosphamide and lymphocytes against melanoma cells. Int J Cancer 2009; 124: 1470-7.

26. Brożyna AA, Van Middlesworth L, Slominski AT. Inhibition of melanogenesis as a radiation sensitizer for melanoma therapy. Int J Cancer 2008; 123: 1448-56.

27. Leary A, Auclin E, Pautier P, Lhommé C. The PI3K/Akt/ mTOR pathway in ovarian cancer: biological rationale and therapeutic opportunities. Ovarian Cancer - A Clinical and Translational Update 2013.

28. Rather MA, Amin S. A comprehensive review on the phytochemical and pharmacological aspects of Podophyllum hexandrum: a high value medicinal plant. Adv Biomed Pharma 2016; 3: 216-26. 